\title{
A MOTION VECTOR RECOVERY ALGORITHM FOR TEMPORAL ERROR CONCEALMENT USING OPTICAL FLOW IN H.264 VIDEO CODING
}

\author{
Donghyung Kim, Sanghyup Cho, and Jechang Jeong \\ Dept. of Electrical and Computer Engineering, Hanyang University \\ Haengdang, Seongdong, Seoul, South Korea
}

\begin{abstract}
For the improvement of coding efficiency, the H.264 standard uses new coding tools. Among the tools, motion estimation using smaller block sizes leads to higher correlation between the motion vectors of neighboring blocks. This characteristic of H.264 is useful for motion vector recovery to conceal a lost macroblock. In this paper, we propose the motion vector recovery method based on optical flow in H.264. We first determine optical flow region to alleviate the complexity, and choose initial value of flow velocity using neighboring motion vectors of a lost macroblock. And then the proposed method recovers the motion vectors of $4 \times 4$ blocks included in a lost macroblock using the weighted average of obtained flow velocities. Simulation results show that our proposed method gives higher objective and subjective visual qualities than conventional approaches.
\end{abstract}

\section{INTRODUCTION}

When packetized video streams are transmitted through noisy channel, channel noise or congestion often leads to packet loss. This can drastically degrade the visual quality of the decoded sequence. As a way to solve this problem, error concealment is very useful, since the decoded frame having lost blocks still includes spatial and temporal redundancy. In temporal error concealment, correlation between current decoded frame and previous decoded frames is exploited. A damaged macroblock of the current decoded frame is replaced by a macroblock in the reference frame using the estimated motion vector of the lost macroblock.

Among conventional approaches for temporal error concealment, the simplest way is a temporal replacement (TR) method which conceals a lost macroblock with a macroblock located at the same position in a previous frame. A TR method produces reasonably good visual qualities in stationary areas, but significant degradations in dynamic areas.

Boundary matching algorithm (BMA) is one of the most popular methods for motion vector recovery. It exploits the fact that adjacent pixels in a video frame exhibit high spatial correlation. The reference software of H.264 also uses a temporal error concealment method based on BMA.
Several approaches to temporal error concealment have been proposed to enhance the performance. Chen et al. proposed the so-called refined boundary matching algorithm (RBMA) based on the boundary matching algorithm [1]. To better satisfy the criterion of minimizing the boundary differences between a lost macroblock and a replacing macroblock in the reference, they use different motion vectors for different regions of a lost macroblock. Zheng, et al. proposed a temporal error concealment method for H.264 by using the Lagrange interpolation formula to constitute a polynomial that describes the motion tendency of motion vectors [2]. It exploits the fact that there is higher correlation between the motion vectors of adjacent blocks in H.264. Suh, et al. proposed the motion vector recovery method using optical flow in MPEG2 [3]. To alleviate the complexity they determine the optical flow region, and recover the motion vector of $16 \times 16$ block by using the average value of flow velocity vectors of neighboring macroblocks. Xu, et al. proposed a set of error concealment schemes to improve error resilience ability for video consumer applications [4]. Their methods include refined motion compensated temporal concealment with weighted boundary matching criterion, an algorithm of refined directional weighted spatial interpolation, and an adaptive spatial/temporal estimation method with low complexity to combine the above algorithms.

In this paper, we propose a motion vector recovery method for temporal error concealment based on optical flow in H.264. Since optical flow fields are very similar to the true motion and can be used to recover threedimensional motion information, we first compute flow velocity vectors by Horn and Schunck's method [5], and then recover the motion vectors of $4 \times 4$ blocks in a lost macroblock using the weighted average of flow velocities.

\section{HORN AND SCHUNCK'S METHOD}

Optical flow is referred to as the two dimension distribution of apparent velocities of movement of intensity patterns in an image plane. In other words, an optical flow field consists of a dense velocity field with one velocity vector for each pixel in the image plane. If we know the time interval between two consecutive images, which is usually the case, then velocity vectors and displacement vectors can be converted from one to another. In this sense, optical flow is one of the techniques used for error concealment. 


\subsection{Constraints for determining optical flow}

To find optical flow, Horn and Schunck use two constraints. They are the brightness invariant constraint and the smoothness constraint.

\subsubsection{Brightness Invariance Constraint}

Let the image brightness at the point $(x, y)$ in the image plane at time $t$ be denoted by $E(x(t), y(t), t)$. If the image brightness is invariant with respect to the time interval from $t$ to $t+\Delta t$, we then have

$$
E(x(t), y(t), t)=E(x(t+\Delta t), y(t+\Delta t), t+\Delta t)
$$

Equation (1) is the brightness invariance equation, strictly speaking, the brightness time-invariance equation. The expansion of the right-hand side of Eq. (1) in the Taylor series at time $t$ leads to

$$
\left(\frac{\partial E}{\partial x} u+\frac{\partial E}{\partial y} v+\frac{\partial E}{\partial t}\right) \Delta t+\varepsilon=0, \quad u=\frac{d x}{d t}, v=\frac{d y}{d t}
$$

where $\varepsilon$ contains second and higher order terms and $u$ and $v$ are the horizontal and vertical components of an optical flow velocity, respectively.

After dividing both sides of the equation by $\Delta t$ and evaluating the limit as $\Delta t \rightarrow 0$, we have a single linear equation in the two unknown parameters: $u$ and $v$.

$$
E_{x} u+E_{y} v+E_{t}=0
$$

where $E_{x}, E_{y}$ and $E_{t}$ are the partial derivatives of image brightness with respect to $x, y$ and $t$, respectively.

Fig. 1 depicts the brightness invariance constraint.

\subsubsection{The Smoothness constraint}

Eq. (3) reveals that we have two unknowns: $u$ and $v$, but only one equation to relate them. It indicates that there is no way to compute optical flow without an additional constraint.

The smoothness constraint means flow velocity vectors to vary from one to another smoothly, particularly for points belonging to the same object. Mathematically, it is imposed in optical flow determination by minimizing the square of the Laplacians of the $x$ and $y$ components of the flow.

$$
(u, v)=\underset{u, v}{\arg \min }\left(\nabla^{2} u+\nabla^{2} v\right)
$$

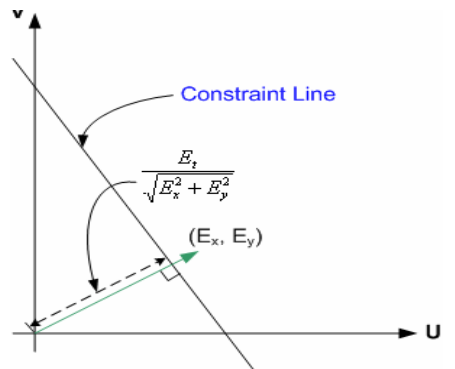

Figure 1. Brightness invariance constraint.

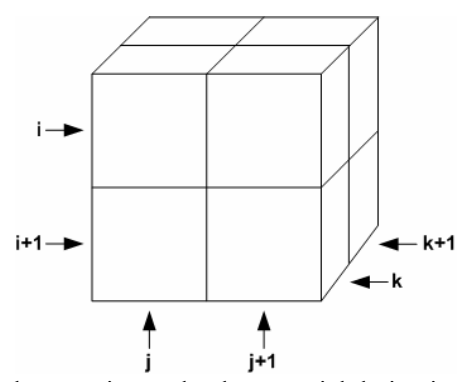

Figure 2. The cube to estimate the three partial derivatives.

\subsection{Approximation}

For determining optical flow using two constraints, we must compute the partial derivatives $\left(E_{x}, E_{y}, E_{t}\right.$ in Eq. (3)) and also the Laplacian of the flow velocities $\left(\nabla^{2} u, \nabla^{2} v\right.$ in Eq. (4)). Horn and Schunck estimate $E_{x}, E_{y}$ and $E_{t}$ at a point in the center of a cube formed by eight measurements as shown in Fig. 2.

Each of the estimates is the average of four first differences taken over adjacent measurements in the cube.

$$
\begin{aligned}
E_{x}=\frac{1}{4} & \left(E_{i, j+1, k}-E_{i, j, k}+E_{i+1, j+1, k}-E_{i+1, j, k}\right. \\
& \left.+E_{i, j+1, k+1}-E_{i, j, k+1}+E_{i+1, j+1, k+1}-E_{i+1, j, k+1}\right) \\
E_{y}=\frac{1}{4} & \left(E_{i+1, j, k}-E_{i, j, k}+E_{i+1, j+1, k}-E_{i, j+1, k}\right. \\
& \left.\quad+E_{i+1, j, k+1}-E_{i, j, k+1}+E_{i+1, j+1, k+1}-E_{i, j+1, k+1}\right) \\
E_{t}=\frac{1}{4} & \left(E_{i, j, k+1}-E_{i, j, k}+E_{i+1, j, k+1}-E_{i+1, j, k}\right. \\
& \left.+E_{i, j+1, k+1}-E_{i, j+1, k}+E_{i+1, j+1, k+1}-E_{i+1, j+1, k}\right)
\end{aligned}
$$

The average can remove the noise effect, thus making the obtained partial derivatives less sensitive to various noises.

The Laplacian of $u$ and $v$ are approximated by

$$
\begin{aligned}
& \nabla^{2} u=\bar{u}_{x, y, k}-u_{x, y, k} \\
& \nabla^{2} v=\bar{v}_{x, y, k}-v_{x, y, k}
\end{aligned}
$$

where $\bar{u}$ and $\bar{v}$ indicate the local average with respect to the $x$ and $y$ components of flow vector, respectively, are estimated as follows

$$
\begin{aligned}
\bar{u}_{x, y, k} & =\frac{1}{6}\left(u_{x-1, y, k}+u_{x, j+1, k}+u_{x+1, y, k}+u_{x, y-1, k}\right) \\
+ & \frac{1}{12}\left(u_{x-1, y-1, k}+u_{x-1, y+1, k}+u_{x+1, y+1, k}+u_{x+1, y-1, k}\right) \\
\bar{v}_{x, y, k} & =\frac{1}{6}\left(v_{x-1, y, k}+v_{x, j+1, k}+v_{x+1, y, k}+v_{x, y-1, k}\right) \\
& +\frac{1}{12}\left(v_{x-1, y-1, k}+v_{x-1, y+1, k}+v_{x+1, y+1, k}+v_{x+1, y-1, k}\right)
\end{aligned}
$$

\subsection{Determining optical flow}

To determine optical flow, the Horn and Schunck method minimizes a weighted sum of the error in two constraints. 


$$
\iint\left(\left(E_{x} u+E_{y} v+E_{t}\right)^{2}+\alpha^{2}\left(\nabla^{2} u+\nabla^{2} v\right)\right) d x d y
$$

In Eq. (8), $\alpha^{2}$ plays a significant role only for areas where the brightness gradient is small, preventing haphazard adjustments to the estimated flow velocity occasioned by noise in the estimated derivatives.

Using the calculus of variations and the approximation of the Laplacians shown in Eq. (6), the minimization of Eq. (8) requires solving the two equations simultaneously.

$$
\begin{aligned}
& \left(\alpha^{2}+E_{x}^{2}+E_{y}^{2}\right)(u-\bar{u})=-E_{x}\left(E_{x} \bar{u}+E_{y} \bar{v}+E_{t}\right) \\
& \left(\alpha^{2}+E_{x}^{2}+E_{y}^{2}\right)(v-\bar{v})=-E_{y}\left(E_{x} \bar{u}+E_{y} \bar{v}+E_{t}\right)
\end{aligned}
$$

Finally, as described in Eq. (10), optical flow can be computed iteratively from the estimated derivatives and the average of the previous velocity estimates by using GaussSeidel method [6]. In this formula, $n$ is iteration number.

$$
\begin{aligned}
& u^{n+1}=\bar{u}^{n}-E_{x}\left(E_{x} \bar{u}^{n}+E_{y} \bar{v}^{n}+E_{t}\right) /\left(\alpha^{2}+E_{x}^{2}+E_{y}^{2}\right) \\
& v^{n+1}=\bar{v}^{n}-E_{y}\left(E_{x} \bar{u}^{n}+E_{y} \bar{v}^{n}+E_{t}\right) /\left(\alpha^{2}+E_{x}^{2}+E_{y}^{2}\right)
\end{aligned}
$$

\section{PROPOSED METHOD}

\subsection{Determining optical flow region (OFR) and choosing the initial value of the flow velocity vector}

Before evaluating the flow velocity vector, we first determine optical flow regions (OFR) which are located at top, bottom, left and right positions of a lost macroblock. Fig. 3 depicts four OFRs used in our method.

Choosing more accurate initial value is very important to have a fast convergence. Since the H.264 standard estimates the motion vector for finer block size, H.264 has higher correlation between motion vectors of adjacent blocks than previous standards. This characteristic of H.264 enables us to choose a good initial value of flow velocity vector using motion vectors adjacent to a lost macroblock.

In our algorithm, four OFRs have the different initial values. We use the average of motion vectors of four $4 \times 4$ blocks adjacent to a lost macroblock as an initial value for each OFR. Fig. 4 indicates the motion vectors for choosing the initial value of velocity vector. Consequently, we can obtain initial values for all OFRs using Eq. (11).

$$
(u, v)_{\text {init }}^{p}=\left(\sum_{i=0}^{3} M V_{i}^{p}\right) / 4 \quad p=T, B, L, R
$$

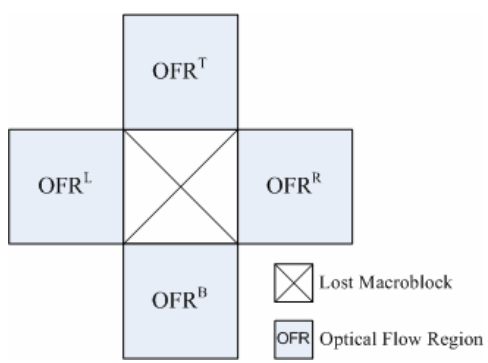

Figure 3. Optical flow region used in our proposed method.

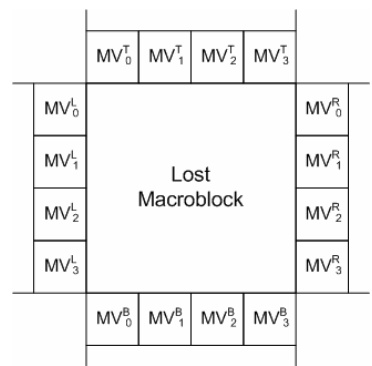

Figure 4. Motion vectors used for choosing the initial value of velocity vector

\subsection{Motion vector recovery}

To obtain optical flow of each OFR, we use iterative scheme described in Eq. (10). After calculation of flow vectors from four OFRs, we take the flow vectors at outer boundaries with one-pixel wide, and recovery motion vectors of $4 \times 4$ blocks using them. Fig. 5 depicts positions of velocity vectors used for motion vector recovery and the recovery ordering. In this figure, $(u, v)_{i}^{T, L, B, R}$ indicates the average velocity vector of four boundary pixels.

For blocks 0 to 3 the process of motion vector recovery is described in Fig. 6. As shown Fig. 6, the top and left flow velocities are used for motion vector recovery in blocks 0 to 3 . In case of block 0 all weights are equal to 1 . In case of block 1 and 2, the different weights are assigned according to distance. And motion vector of block 3 is recovered by using median vector of blocks 0 to 2 . This process can be formulized as Eq. (12).

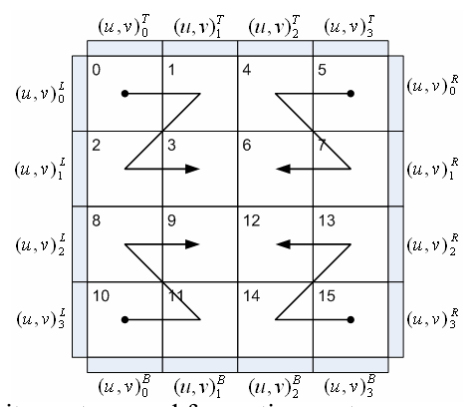

Figure 5. Velocity vectors used for motion vector recovery and the recovery ordering
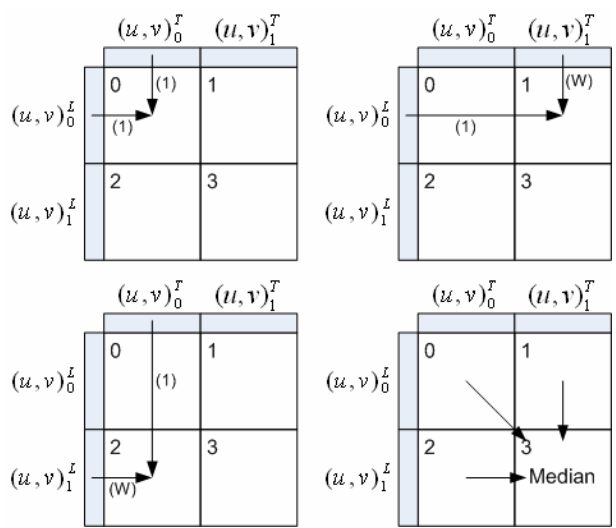

Figure 6. Motion vector recovery of block 0 to $3((\bullet)$ weight $)$ 


$$
\begin{aligned}
& M V_{0}=\left((u, v)_{0}^{T}+(u, v)_{0}^{L}\right) / 2 \\
& M V_{1}=\left(w \cdot(u, v)_{1}^{T}+(u, v)_{0}^{L}\right) /(1+w) \\
& M V_{2}=\left((u, v)_{0}^{T}+w \cdot(u, v)_{1}^{L}\right) /(1+w) \\
& M V_{3}=\operatorname{Median}\left(M V_{0}, M V_{1}, M V_{2}\right)
\end{aligned}
$$

For the other blocks (blocks 4 to15), the same process is used.

\section{SIMULATION RESULTS}

To evaluate the proposed algorithm, we used a public reference encoder, JVT Model (JM) v.10.1 [7]. Three standard video sequences in QCIF $(176 \times 144)$ format are analyzed. These include Coastguard, Foreman, and Table Tennis. The first 100 frames of each sequence are used. We compare the simulation results of the proposed algorithm with those of the temporal replacement, the H.264 reference implementation, and Zheng's method [2]. In our simulation, $n$ was limited below 32 times and $w$ in Eq. (12) was set to 2 .

Figs. 7 to 8 illustrate objective and subjective qualities of the proposed method compared with conventional approaches. As shown in these results the proposed method gives better visual qualities than conventional approaches.

\section{CONCLUSION}

For temporal error concealment in H.264 we propose the motion vector recovery technique based on optical flow. We first determine four optical flow regions, and calculate flow vectors from them. And then the proposed method recovers the motion vectors of $4 \times 4$ blocks in a lost macroblock by weighted averaging of flow vectors. Simulation results show that the proposed method outperforms conventional methods in terms of subjective and objective video quality.

\section{ACKNOWLEDGEMENT}

This research was supported by Seoul Future Contents Convergence (SFCC) Cluster established by Seoul IndustryAcademy-Research Cooperation Project.

\section{REFERENCES}

[1] T. Chen, X. Zhang, and Y. Q. Shi, "Error Concealment Using
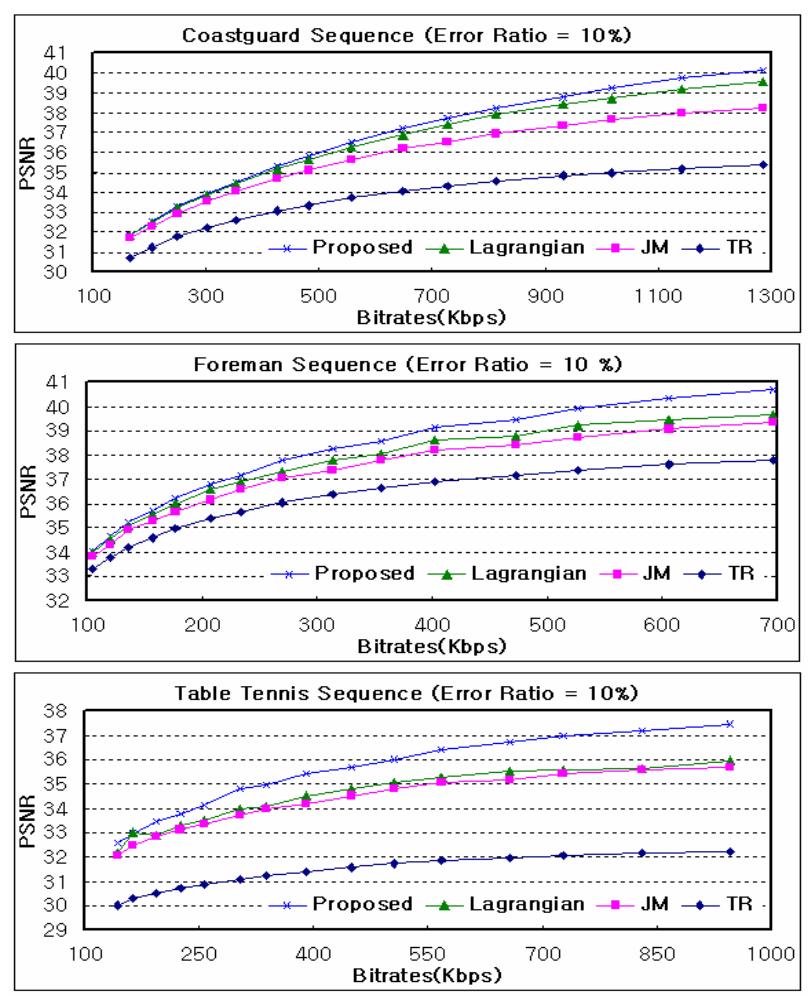

Figure 7. Comparison of objective video qualities when there is block error ratio of $10 \%$

Refined Boundary Matching Algorithm," IEEE Trans. Circuits and Systems for Video Technology, vol. 13, pp. 560-576, July 2003. [2] J. Zheng and L.P. Chau, "A Motion Vector Recovery Algorithm for Digital Video Using Lagrange Interpolation," IEEE Trans. Broadcasting, vol. 49, pp. 383-389, Dec. 2003.

[3] J.W. Suh and Y.S. Ho, "Error Concealment Technique for Digital TV," IEEE Trans. Broadcasting, vol. 48, pp. 299-305, Dec. 2002.

[4] Y. Xu and Y. Zhou, "H.264 Video Communication Based Refined Error Concealment Schemes," IEEE Trans. Consumer Electronics, vol. 50, pp. 1135-1141, Nov. 2004.

[5] B.K.P. Horn and B.G. Schunck, "Determining optical flow," Artificial Intelligence, vol. 17, pp. 185-203, 1981.

[6] A. Tekalp, Digital Video Processing, Prentice Hall, 1995.

[7] JM 10.1: http://bs.hhi.de/ suehring/tml/download/jm101.zip.

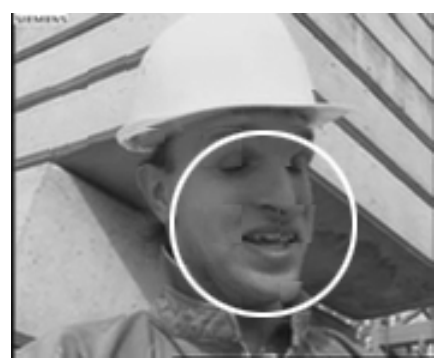

(a)

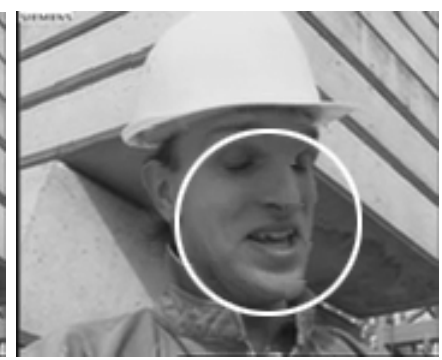

(b)

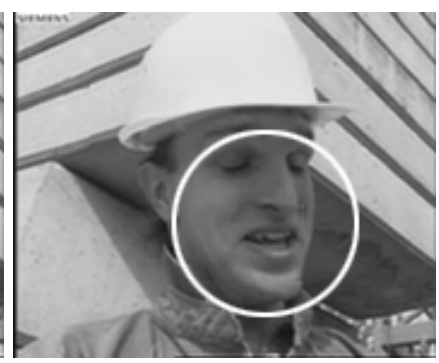

(c)

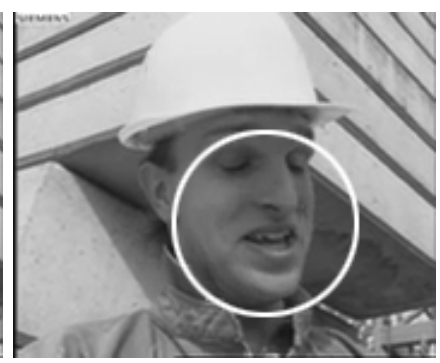

(d)

Figure 8. Comparison of subjective video qualities for the $76^{\text {th }}$ frame of the foreman sequence (a) temporal replacement (b) reference implementation [7] (c) Zheng's method [2] (d) the proposed method 